\title{
OH maser survey of very cool IRAS sources
}

\author{
P. te Lintel Hekkert and A. Heske \\ Sterrewacht Leiden \\ P.O. Box 9513, 2300 RA Leiden, The Netherlands \\ A.M. Le Squeren \\ Observatoire de Paris \\ Section de Meudon, DERADN, F-92190, Meudon, France
}

During our $1612 \mathrm{MHz}$ survey of IRAS sources in Parkes, Australia, we found two objects with very large expansion velocities : IRAS15405-4945 and IRAS163423814 (P. te Lintel Hekkert et al. 1988, Astron. Astrophys. (Letters), in press.). These two sources and the well known source, OH231.8+4.2, were discovered to have almost identical IRAS colours. The spectra of the three sources could be fitted with a $100( \pm 15) \mathrm{K}$ black body curve. We than selected, using the IRAS colour-colour plot, a number of bright sources ( F $(60 \mu \mathrm{m})>25$. Jy ) with similar colours to OH231.8 +4.2 and IRAS16342-3814 . A small survey of about 150 sources was started in the last week of June and will be finished at the end of July 1988. Using the radio telescope in Nançay, we observed all four $\mathrm{OH}$ maser transitions, with the hunderd meter radio telescope at Effelsberg only $1612 \mathrm{MHz}$ was observed, the sources found at Nançay were confirmed in Bonn and vice versa. From the preliminary analyses of the first $\mathbf{4 0}$ observations six sources were found of which three were not known before.

IRAS22036 +5306 shows the characteristic two spike profile of OH/IR stars at the $1612 \mathrm{MHz}$ and $1667 \mathrm{MHz}$ transitions. This source has very red colours compared with "regular" OH/IR stars. Most likely this is an example of an $\mathrm{OH} / \mathrm{IR}$ star which has stopped it's mass loss several hunderd years ago. Since than the original CSE has expanded and cooled. The presence of the $1667 \mathrm{MHz}$ could also be interpret in this way (B.M. Lewis 1988, preprint). It has been suggested by several authors (e.g. J. Sun and S. Kwok 1987, Astron. Astrophys. $185,285)$ that these cool OH/IR stars are PPN's.

The other two were found to have $1667 \mathrm{MHz}$ emission only. IRAS174231755 showed a profile similar to the one of OH231.8+4.2: a wide plateau of emission on top of which there is a broad spike. The profile of IRAS184910207 resembles the one of IRAS16342-3814. : two bunches of spikes, with a total velocity extent of $140 \mathrm{~km} \cdot \mathrm{s}^{-1}$ ! The shape of the $\mathrm{OH}$ spectra suggest that the maser emission is unsaturated and pumped by photons from the high energy background (the hot star or nebula). The differences in shape of the profiles of the sources can be explained by assuming different orientation of the axis joining the two reflection nebulae. The high expansion velocity and the probable existance of the hot component in the system suggests that these are short lived stages of stellar evolution, most likely PPN's. 